\title{
Imaging of coronavirus disease (COVID-19): a pictorial review
}

\author{
Ayush Jain ${ }^{A, B, D, E, F}$, Soniya Patankar ${ }^{A, B, D, E, F}$, Sunita Kale ${ }^{A, D, E}$, Abhishek Bairy ${ }^{A, B, D, E, F}$ \\ Department of Radiology, Seth G.S. Medical College \& K.E.M. Hospital, Mumbai, Maharashtra, India
}

\section{Abstract}

\begin{abstract}
Coronavirus disease (COVID-19), caused by a highly contagious novel coronavirus, has seen a rapid surge of cases over the past 6 months spreading to more than 215 countries and posing a global threat to mankind. Reverse transcriptase-polymerase chain reaction (RT-PCR) from pharyngeal swabs is considered the gold standard for diagnosis of this disease. Portable chest radiography (CXR), point of care ultrasound, and computed tomography (CT) are crucial modalities in diagnosis and follow-up. Portable CXR can help in patients who are clinically unstable, and also to prevent the cumbersome process of steriliastion after every CT scan. However, chest CT is useful as a problem-solving tool, to look for progression and complications associated with the disease. In a few cases, in our experience (as has also been documented by others), RT-PCR was negative in early disease, and CT chest was able to detect the radiological findings raising suspicion of COVID-19. With this pictorial review, we aim to describe and illustrate the typical, and a few atypical, radiological findings of this disease.
\end{abstract}

Key words: computed tomography, pneumonia, coronavirus, radiograph, ground glass opacity, ARDS.

\section{Introduction}

Coronavirus disease 2019 (COVID-19), an on-going pandemic caused by severe acute respiratory syndrome coronavirus 2 (SARS-CoV-2), was reported for the first time in Wuhan city of Hubei province in China in December 2019. It has since spread rapidly to other parts of the world, causing a great deal of economic, social, historic, and scientific impact [1]. The World Health Organisation (WHO) declared the outbreak a global public health emergency on January $30^{\text {th }}, 2020$.

The clinical symptoms of COVID-19 can range from asymptomatic to mild disease (generalised body ache, dry cough, fever, and nasal congestion). The severe form of the disease is characterised by hypoxaemia, septic shock, coagulopathy, metabolic abnormalities, and death [2]. Altered sense of smell or taste in mild stages of the disease has also been observed [3]. Laboratory abnormalities include leucopaenia, elevated levels of serum ferritin, altered liver function tests, and raised cytokine levels (IL-6, IL-10). Evidence shows that a cytokine storm is induced by the virus by over-activation of the T cells, which causes lung damage leading to acute lung injury (ALI) [4].

The diagnosis of COVID-19 pneumonia is made by the detection of viral nucleic acid in pharyngeal swabs, sputum, or blood. The specificity of this test is high, with low sensitivity, due to which few patients show false negative results [5]. Studies have shown that radiological findings are observed earlier than the symptoms, which makes imaging important for early diagnosis and timely isolation [6]. This pictorial review aims to highlight the chest radiography (CXR) and computed tomography (CT) patterns of lung abnormality and common manifestations of COVID-19 pneumonia. A brief mention of point-ofcare ultrasound (POCUS) has also been made. An awareness of these features will better equip radiologists in making an early and appropriate diagnosis.

\section{Imaging in COVID-19}

CXR and CT form the mainstay for imaging diagnosis of COVID-19 pneumonia.

Correspondence address:

Dr. Abhishek Bairy, Department of Radiology, Seth G.S. Medical College \& K.E.M. Hospital, Acharya Donde Marg, Parel East, Parel, Mumbai, Maharashtra 400012, India, e-mail: abhishekbairy@gmail.com

Authors' contribution:

A Study design · B Data collection · C Statistical analysis · D Data interpretation - E Manuscript preparation · F Literature search · G Funds collection 


\section{Chest radiographs}

CXR have a vital role to play in COVID-19 pneumonia, considering its highly contagious nature. Due to infection control issues and the requirement of effective decontamination of CT suites after scanning every patient, CT may not be possible for all patients. Furthermore, CXR is more readily available, inexpensive, and is the initial modality for screening [7]. In severe disease with acute respiratory distress syndrome (ARDS), portable CXRs are of paramount importance because patients cannot be mobilised for CT. A few specific patterns of disease have been observed, which are described below.

\section{Ground-glass opacities}

Ground-glass opacities are the earliest and the most common imaging manifestation of COVID-19 pneumonia; however, CXR has a low sensitivity for detection (69\%) [8]. They become more conspicuous when an accompanying reticular pattern is present.

\section{Peripheral alveolar opacities (Figure 1)}

The predilection of COVID-19 to affect the peripheral lung fields is what makes it unique from other types of

A
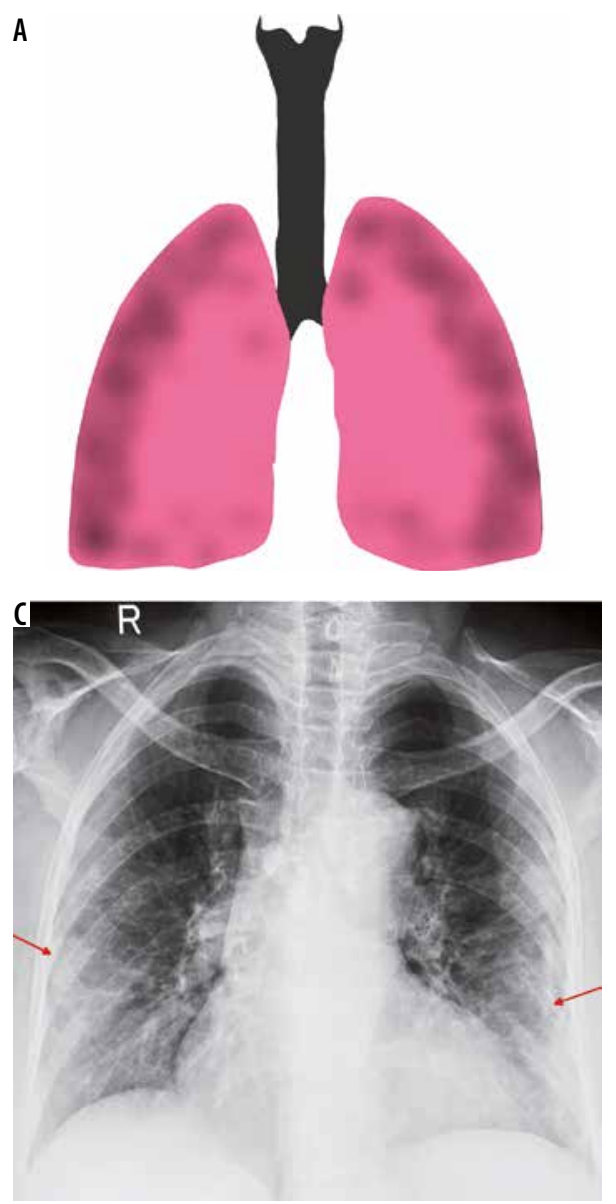

pneumonia. These peripheral opacities may be patchy or confluent and can be picked up on chest radiographs [9]. The opacities in COVID-19 pneumonia are often bilateral and multifocal, as compared to bacterial pneumonia, which is often unilateral and limited to a single lobe [7].

\section{Lower lobe predominant consolidation (Figure 2)}

COVID-19 and other viral pneumonias typically present as lung opacities involving more than one lobe with a propensity for lower lung involvement $[7,9]$. A unilateral, central, or upper lobe predominant distribution is suspicious but indeterminate for COVID-19 infection and can also be seen in a variety of other infectious and noninfectious conditions.

\section{Multifocal and diffuse air space opacities (Figures 3 and 4)}

Diffuse air space opacities in COVID-19 are similar to the ARDS-like diffuse process that is seen with other widespread pulmonary infections. Most patients with these patterns are severely hypoxic and are usually intubated or on mechanical ventilation. The appearance is that of multifocal diffuse alveolar opacities in both lung fields and is usually seen in the late first or early second week of clinical symptoms [7].
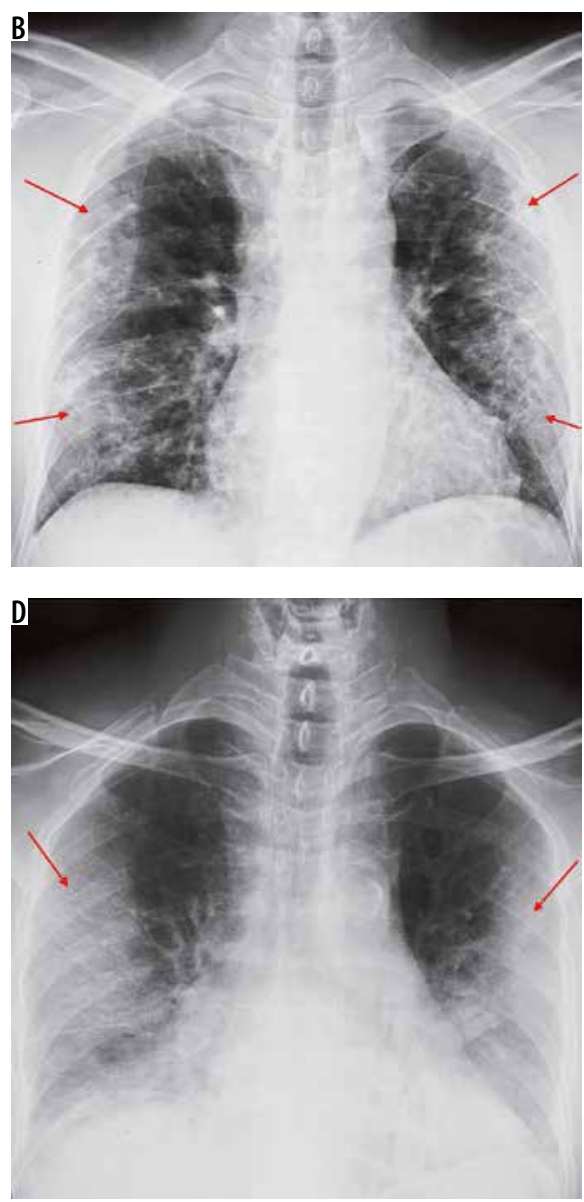

Figure 1. Peripheral predominant pattern of COVID-19 pneumonia. A) Schematic diagram depicting a peripheral predominant pattern of disease. B-D) Frontal chest radiography of 3 different patients with COVID-19 show peripheral, reticular, and patchy alveolar opacities (red arrows) in both lung fields 
A
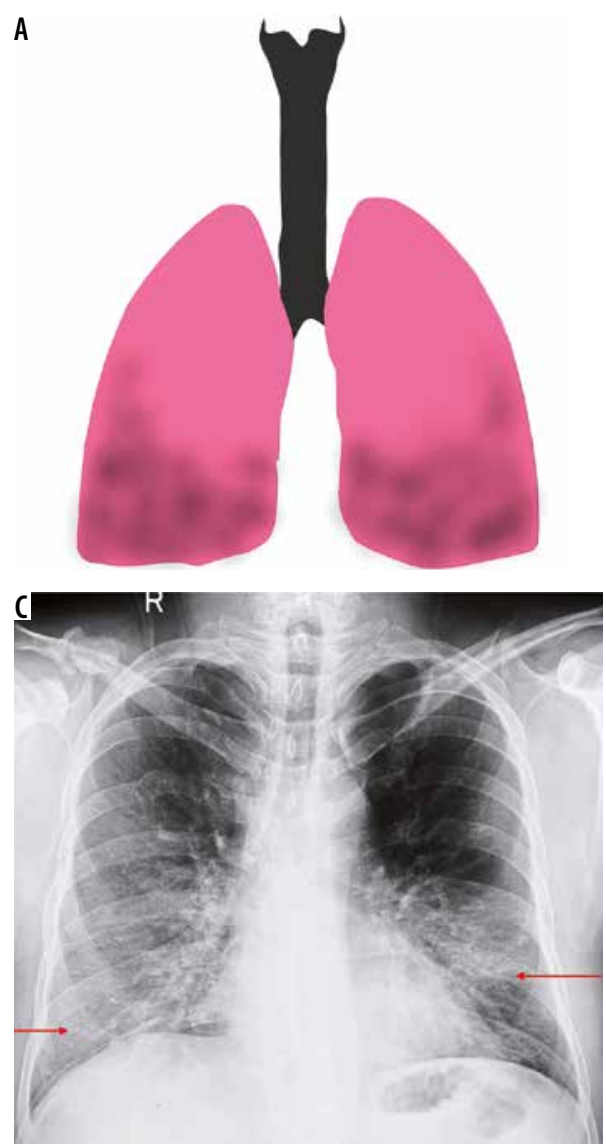
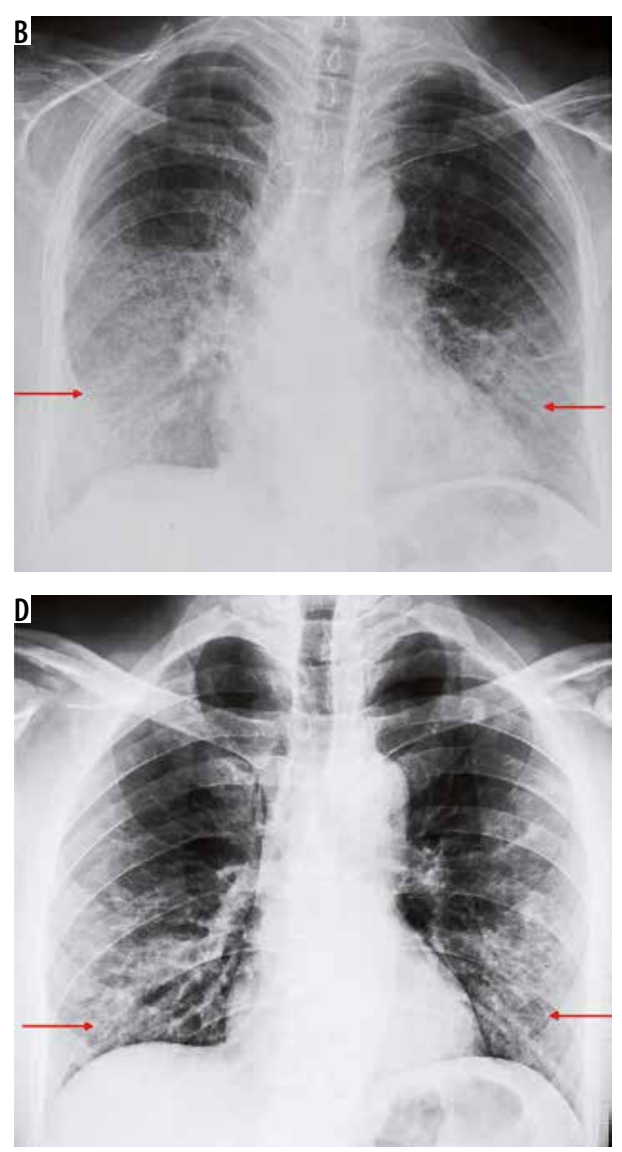

Figure 2. Lower lobe predominant pattern of COVID-19 pneumonia. A) Schematic diagram depicting a lower lobe predominant pattern of disease. B-D) Frontal chest radiography of 3 different patients with COVID-19 show patchy opacities with lower lobe predominance (red arrows)

A
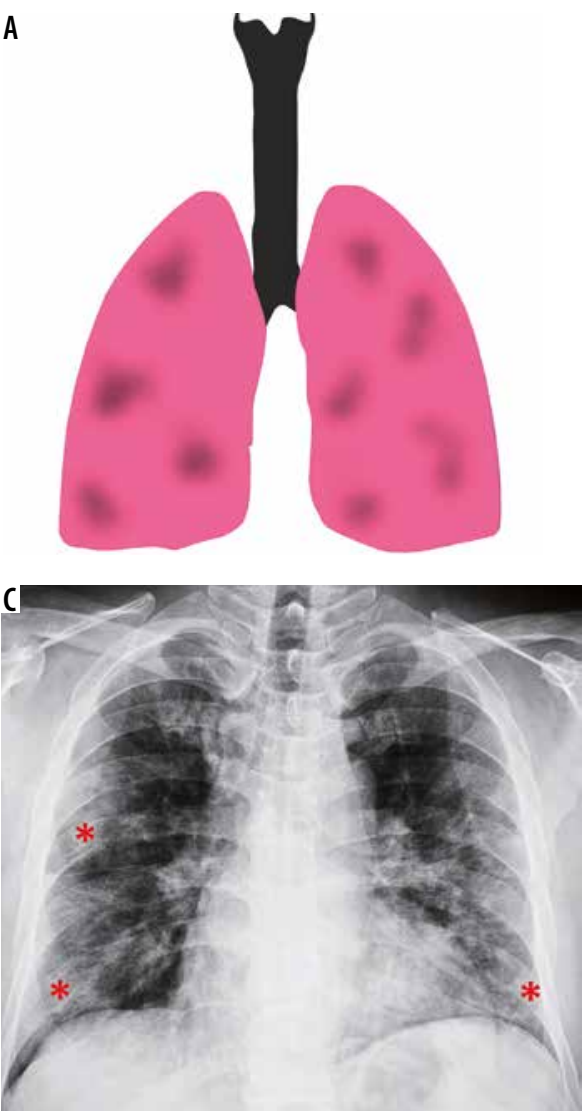
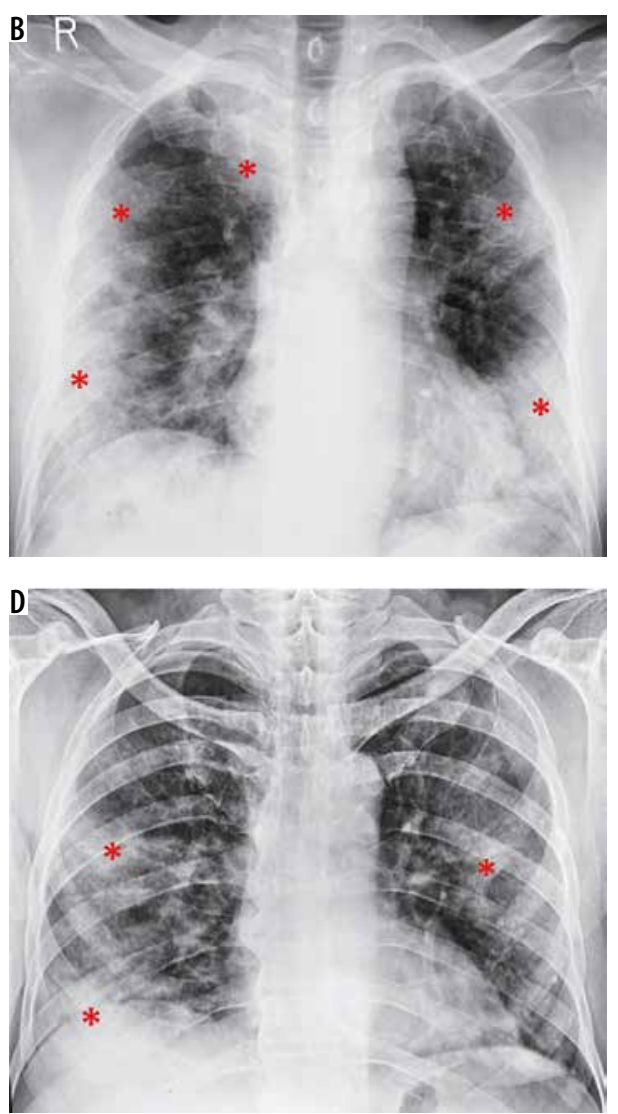

Figure 3. Multifocal pattern of (OVID-19 pneumonia. A) Schematic diagram depicting a multifocal pattern of disease. B-D) Frontal chest radiography of 3 different patients with COVID-19 show areas of patchy alveolar opacities distributed in both lung fields (red asterisks) 

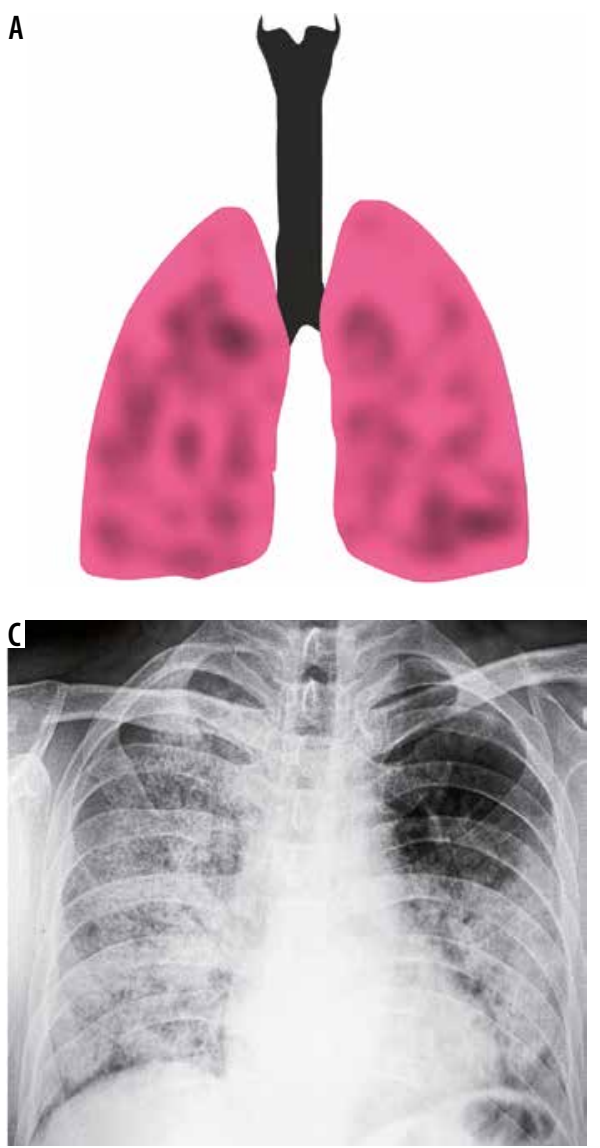
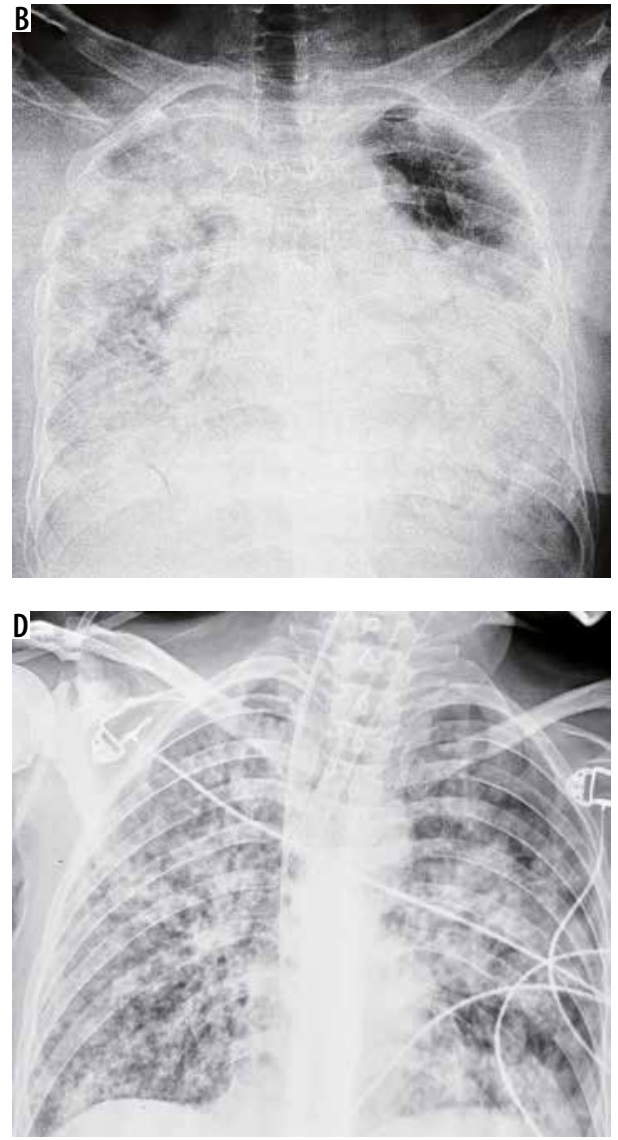

Figure 4. Diffuse pattern of COVID-19 pneumonia. A) Schematic diagram depicting a diffuse pattern of disease. B-D) Frontal chest radiography of 3 different patients with clinical diagnosis of acute respiratory distress syndrome due to COVID-19 show varying degrees of parenchymal opacification in both lung fields

A caveat to this is that a normal CXR is not uncommon in an asymptomatic individual or those presenting early in the course of the disease. Hence, the role of CT scan of the chest in COVID-19 infection is indispensable. In our experience, few patients had imaging findings of COVID-19 with an initial negative swab, as illustrated in Figure 5.

\section{Point-of-care ultrasound}

Ultrasound (US), being a readily available and dynamic modality, is relevant in the imaging of COVID-19 pneumonia. Due to the high contagiousness of the disease and inability to transport unstable patients from the intensive care unit, CT may present as a limited option. In these settings, POCUS may provide results similar to CT and superior to radiographs in evaluation and follow-up [10].

US is performed by placing curvilinear or linear highfrequency transducers along the intercostal spaces. On US, findings such as thickening of the pleural line, B lines (appearing as multiple hyperechoic laser-like reverberation artefacts extending from the pleural line to the base of the screen without fading), and hypoechoic lung with air bronchograms due to alveolar consolidation have been described in cases of COVID-19 pneumonia [10,11]. Few such findings are demonstrated in Figure 6.

\section{Computed tomography}

SARS CoV-2 utilises the angiotensin-converting enzyme-2 (ACE-2) receptors in humans and causes damage to the pulmonary interstitium and subsequently the parenchyma [12]. Although a pharyngeal swab test is the standard assessment for 2019-nCoV infection diagnosis, current tests are time-consuming and may not be easily available due to the high demand. Sampling errors or insufficient viral material in the specimen may also be responsible for a false negative test result.

The critical role of CT in the early detection of patients with COVID-19 infection has modified treatment protocols in many parts of the world. Non-contrast CT obtained in the supine position with breath-hold to minimise motion artifacts is the standard protocol in many institutes for diagnosis of COVID-19 infection [13]. At our institution, high-resolution computed tomography (HRCT) scans were performed with all patients in the supine position at end inspiration. Use of intravenous contrast was limited to patients with suspected pulmonary thromboembolism and raised D-dimer levels. All images were reconstructed at $1.0 \mathrm{~mm}$ slice thickness with $1 \mathrm{~mm}$ increment, $512 \mathrm{~mm}$ $\times 512 \mathrm{~mm}$, and a sharp reconstruction kernel.

Various CT appearances of COVID-19 have been described in the literature, which will be illustrated in more 

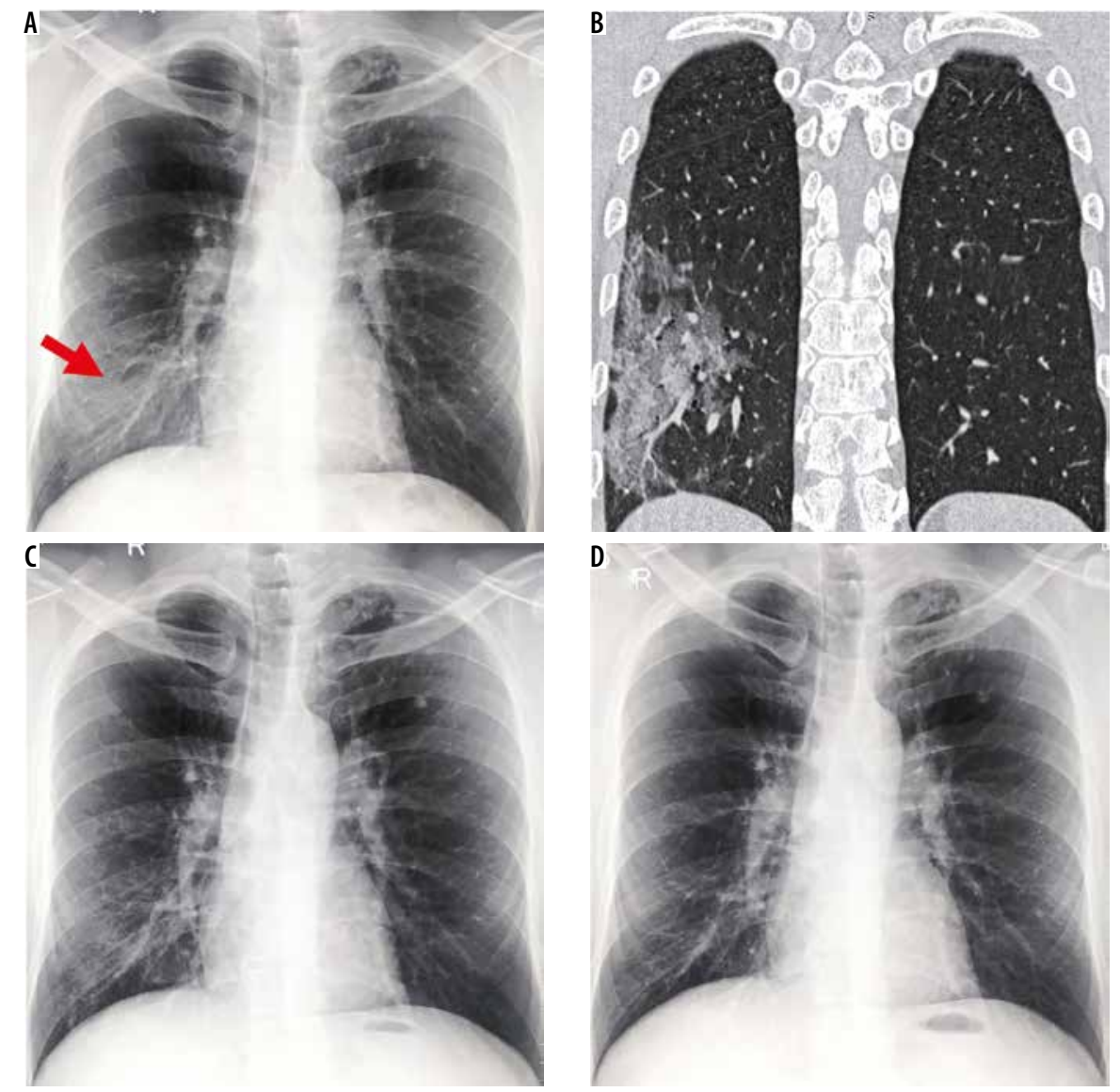

Figure 5. A 50-year-old man with COVID-19 pneumonia came with high-grade fever and loss of appetite for 2 days. The initial nasopharyngeal swab was negative for SARS-CoV-2. However, (A) frontal chest radiography on day 1 of presentation shows subtle ill-defined patchy opacity in the right lower zone (red arrow). B) Coronal reformatted computed tomography chest image shows a peripheral area of ground-glass opacity with interlobular septal thickening (crazy-paving appearance) in the right lower lobe. Follow-up radiographs on day 14 (C) and day 28 (D) showed subsequent resolution of the findings
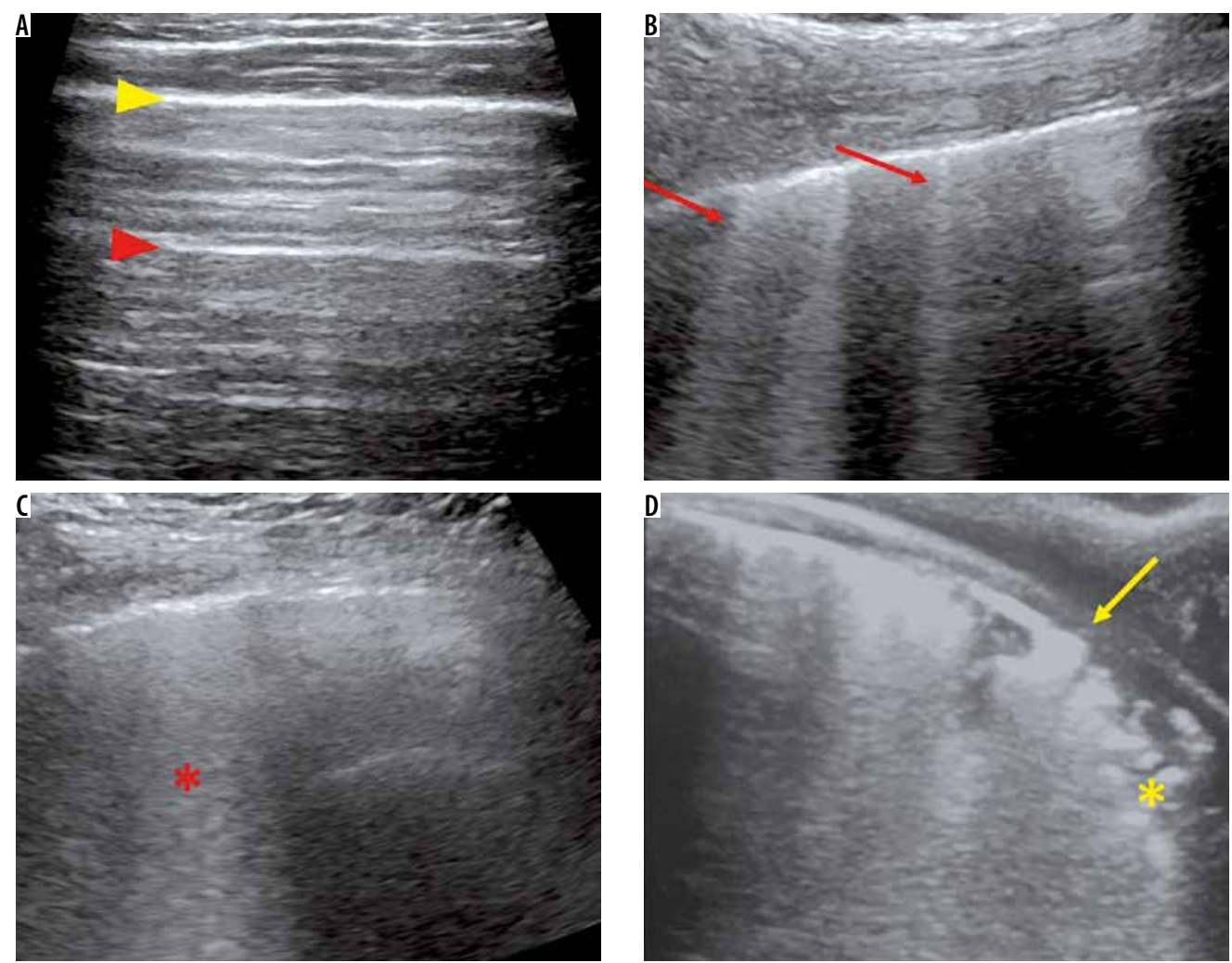

Figure 6. Ultrasound (US) findings in COVID-19 pneumonia. A) Grey-scale US image of the normal lung using a linear array transducer shows a continuous thin pleural line (yellow arrowhead) with multiple reverberation artefacts parallel to it - A lines (red arrowhead). B) A 54-year-old man with COVID-19 pneumonia. US performed using a curvilinear array transducer shows multiple laser-like hyperechoic lines erasing the A lines - B lines (red arrows). C) Confluent B-lines in the same patient as (B) - Waterfall sign (red asterisk). D) A 62-year-old woman with COVID-19 pneumonia. US performed using a linear array transducer showing thickening and interruption of the pleural line (yellow arrow) with subpleural consolidation (yellow asterisk) 
detail. The "Expert Recommendations from the Chinese Medical Association Radiology Branch" divides the CT manifestations of COVID-19 into three stages: early, advanced, and severe, based on the extent of lung involvement with an additional dissipation stage [14].

\section{Early stage (0-4 days)}

Ground-glass opacity (GGO) (Figures 7 and 8) is defined as increased lung opacity that does not obscure the vascu- lar margins. It is caused by partial filling of air spaces with fluid, pus, or blood and resultant displacement of air [15]. The occurrence of multifocal unilateral or bilateral rounded GGOs in the periphery of the lung fields with a subpleural predominance is a common finding in patients with COVID-19 pneumonia. Multiple studies have confirmed these findings with accompanying features of interlobular septal thickening, fibrosis, and consolidation $[16,17]$.

Vascular enlargement (Figure 9) is the dilatation of the pulmonary vasculature within or around the lesions
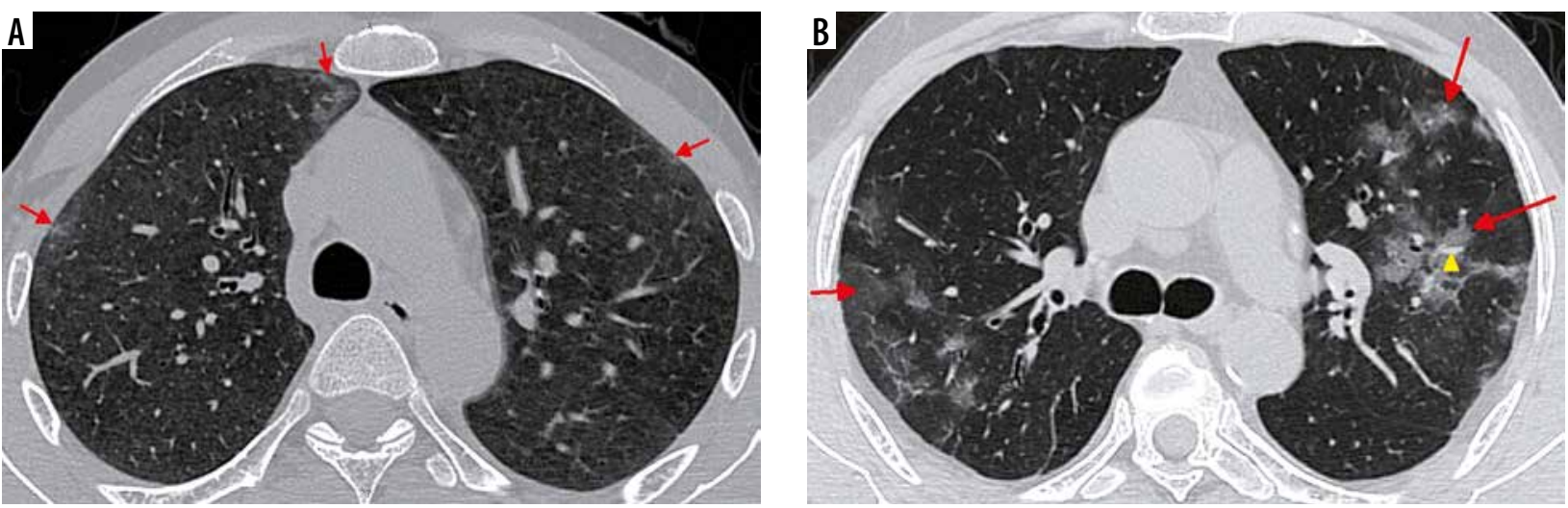

Figure 7. Distribution of ground-glass opacities on axial sections of high-resolution computed tomography chest in 2 different patients with COVID-19 pneumonia. A) A 37-year-old man, 3 days after symptom onset - subtle GGOs in the periphery of both lung fields (red arrows). B) A 49-year-old man, 5 days after symptom onset - multifocal GGOs (red arrows) with vascular thickening (yellow arrowhead) in both lung fields
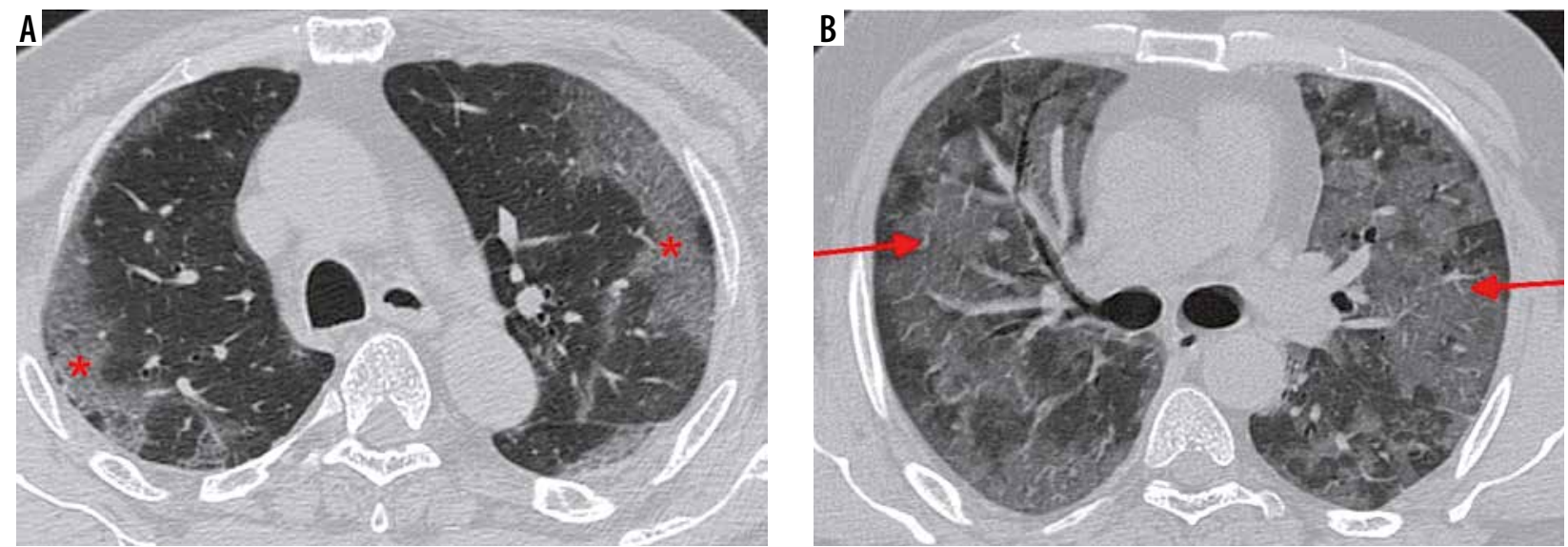

Figure 8. Axial high-resolution computed tomography (HRCT) chest images. A) Confluent ground-glass opacities with a peripheral and subpleural distribution in both lung fields (red asterisks) in a 47-year-old woman with significant hypoxia. B) A 52-year-old man presented to the emergency room with breathlessness and poor oxygen saturation for three days. Axial HRCT image shows extensive, diffuse GGOs in both lung fields (red arrows) with few areas of spared lung parenchyma. Nasopharyngeal swabs in both patients confirmed COVID-19 pneumonia
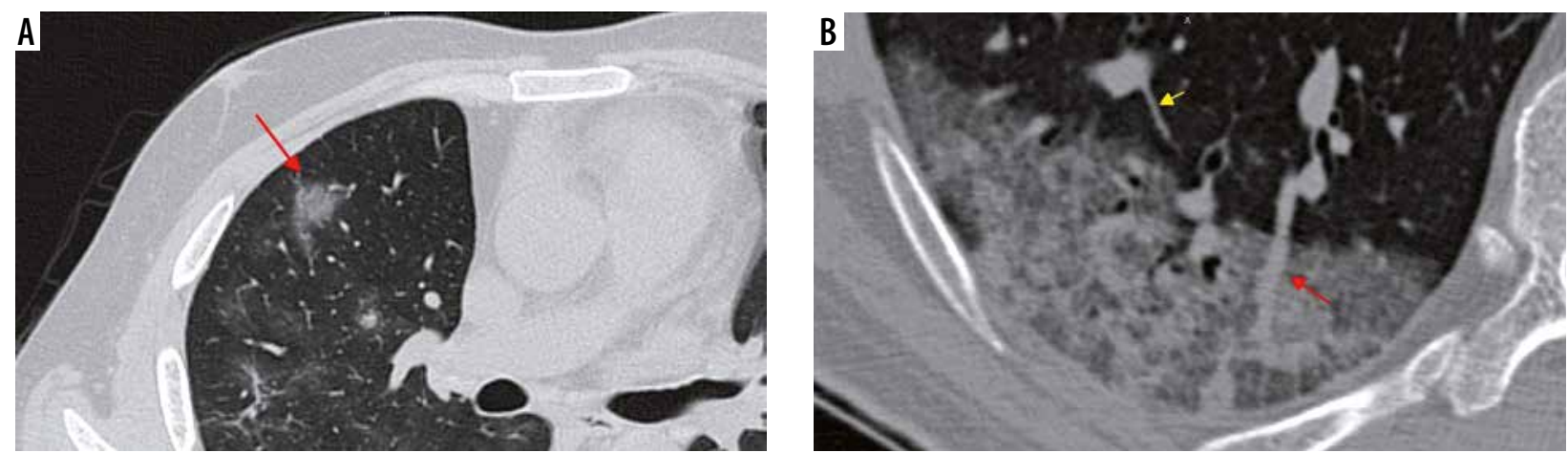

Figure 9. A) Coned down axial high-resolution computed tomography chest image of a patient with COVID-19 shows rounded ground-glass opacity (GGO) in the right upper lobe (red arrow). B) Coned down axial computed tomography image in a different patient shows vascular thickening (red arrow), in a background of interlobular septal thickening with GGOs (crazy paving appearance). The normal calibre of a peripheral vessel is shown for comparison (yellow arrow) 
seen on CT. This manifestation may be due to endothelial damage to the capillary walls resulting from inflammatory mediator release.

\section{Advanced stage (5-8 days) (Figure 10)}

Chest CT in this stage shows new focal lesions with an increase in the density of the previous GGOs, resulting in consolidations with air bronchograms.

Crazy paving pattern (Figure 10A): Thickening of the interlobular septa with superimposed GGOs is known as
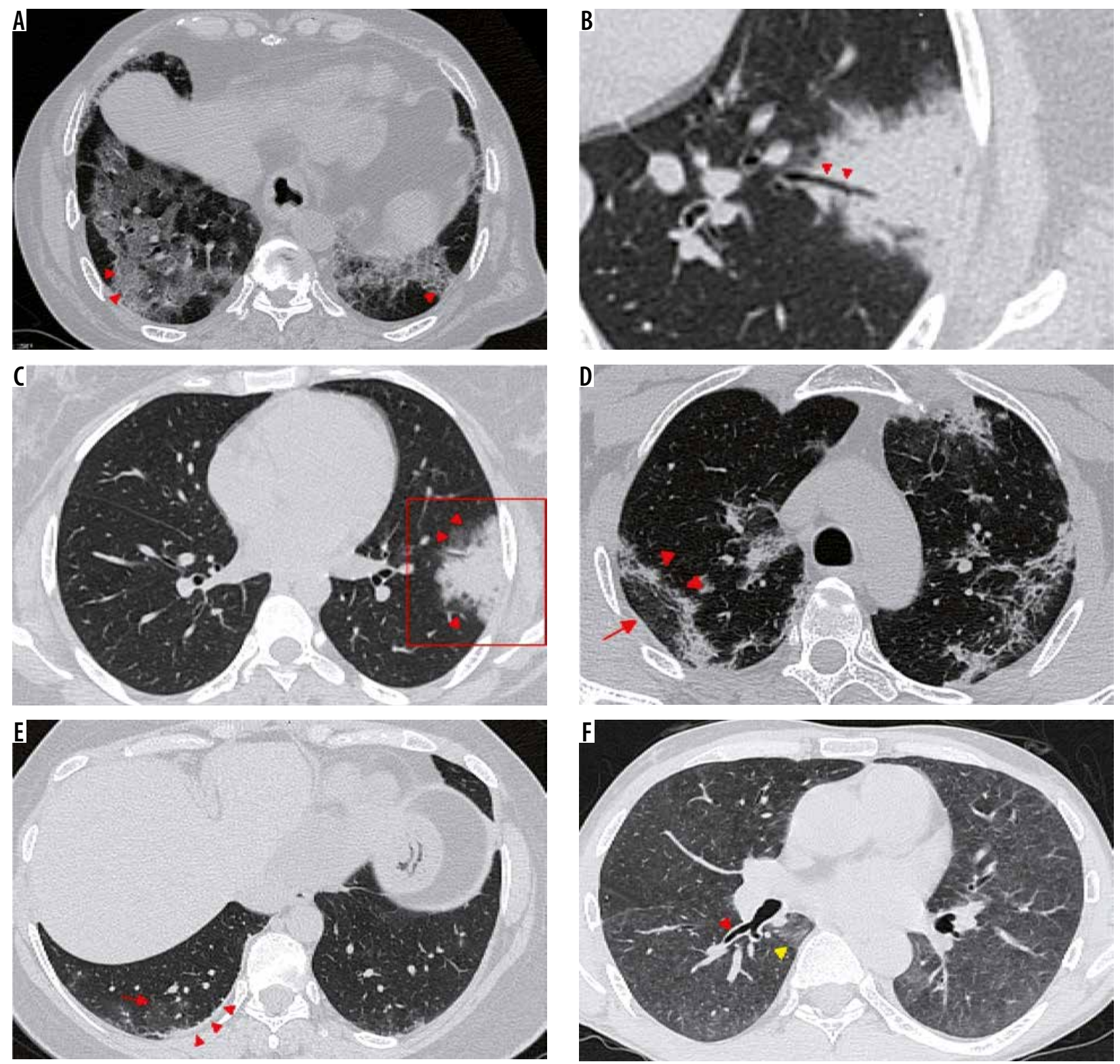

Figure 10. Axial thin sections of chest high-resolution computed tomography (HRCT) of patients with COVID-19 pneumonia in first and the second week of symptoms. A) A 50-year-old man with ground-glass opacities (GGOs) and interlobular septal thickening giving a crazy paving appearance in the superior segment of the right lower lobe (red arrowheads). B) Coned down axial HRCT chest image shows patchy consolidation with a traversing patent bronchus - air bronchogram (red arrowheads) in the left lower lobe of a 26-year-old female presenting with high-grade fever. The surrounding GGO gives the appearance of a halo (halo sign). This is better depicted in (C) obtained at another level (red arrowheads). D) A 37-year-old female with COVID-19 pneumonia. HRCT chest obtained 9 days after symptom onset, shows perilobular consolidation (red arrowheads) with reticular densities in both upper lobes giving rise to a reverse halo - atoll sign (red arrow). E) Lower sections of the chest in the same patient as (D) shows ill-defined GGOs in the posterobasal segment of the right lower lobe (red arrow) with adjacent pleural thickening (red arrowheads). F) Bronchial wall thickening (red arrowhead) in a 40-year-old male. Note the subtle GG0 in the medial basal segment of the right lower lobe (yellow arrowhead) 
patchy consolidations with a peripheral and subpleural predominance in a peribronchovascular distribution is another common presentation of COVID-19 pneumonia. It is thought to occur due to cellular and fibro-myxoid exudates within the alveoli [19]. The role of positron emission tomography/ computed tomography (PET/CT) in COVID-19 pneumonia has been sparsely documented in the published literature with few reports of 18-fluoro-deoxy-glucose (FDG) avidity within the consolidations and associated mediastinal lymph nodes, similar to Middle East respiratory syndrome (MERS) or H1N1 influenza virus $[20,21]$.

Halo and reverse halo signs (Figure 10C-D): Halo sign refers to nodules surrounded by GGO [15]. This sign is seen in hyper-vascular metastasis and angio-invasive fungal infections due to perilesional haemorrhage. It has also been described in atypical infections and organising pneumonia [22], the pathophysiology of which is unclear.

The reverse halo or atoll sign is defined as a focal rounded GGO or normal lung parenchyma surrounded by a ring of consolidation [15].

Pleural changes (Figure 10E): Pleural thickening and pleural effusion have rarely been reported in the disease, with pleural thickening being relatively more common [23].
The appearance of pleural effusion may suggest a poorer prognosis in COVID-19, similar to the findings in the Middle East respiratory syndrome corona virus (MERSCoV) [23].

Airway changes (Figure 10F): Bronchiectasis and bronchial wall thickening have been reported in multiple cases; these findings are likely to be due to inflammatory damage to the bronchial walls followed by fibrosis, scarring, and resultant traction bronchiectasis $[17,24]$.

Air bubble sign (Figure 11): A section through an area of bronchiolectasis or partial resorption of consolidation may give rise to central lucencies, which are referred to as air bubble sign [23].

\section{Severe stage (9-13 days) (Figure 12B)}

This stage shows diffuse multifocal consolidations with air bronchograms and bronchial dilatation.

\section{Dissipation stage ( $\geq 14$ days) (Figure 13)}

This stage is seen most commonly after two weeks and is characterised by gradual resolution of the GGOs and consolidations with residual fibrosis and curvilinear lines.
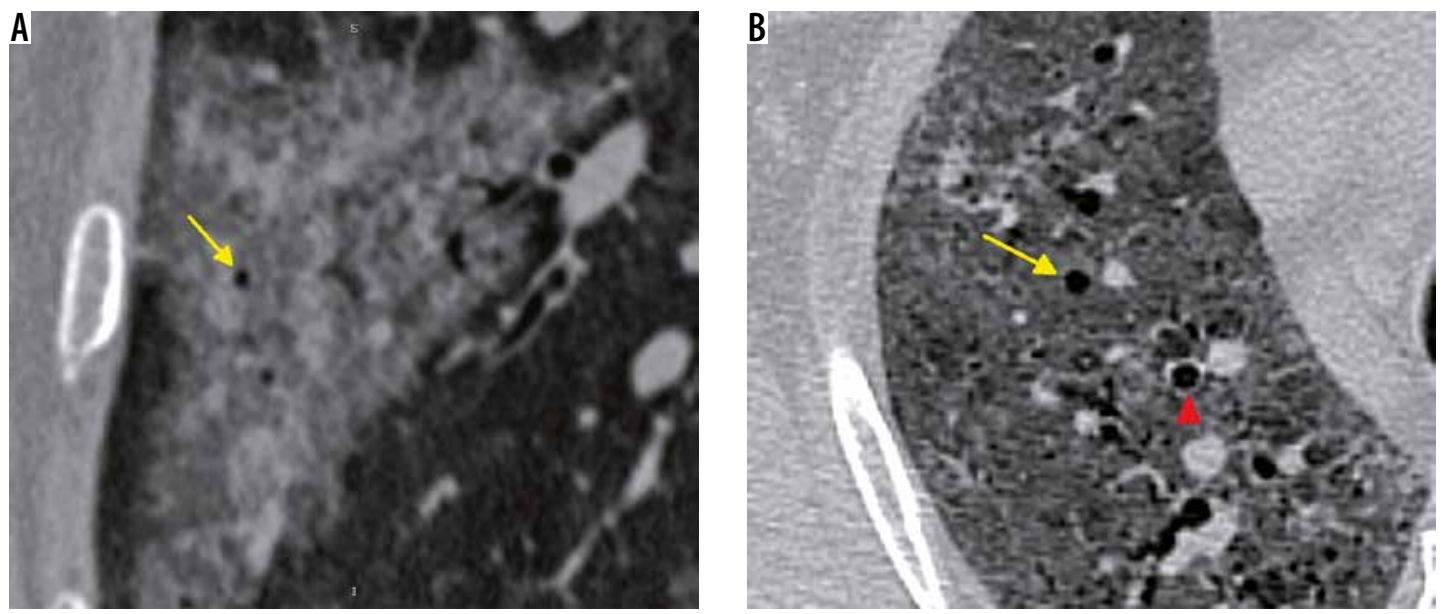

Figure 11. Rounded lucencies within an area of ground-glass opacity in 2 different patients of COVID-19 pneumonia - air bubble sign (yellow arrows). These lucencies have an imperceptible wall, which differentiates them from a normal bronchus (red arrowhead)
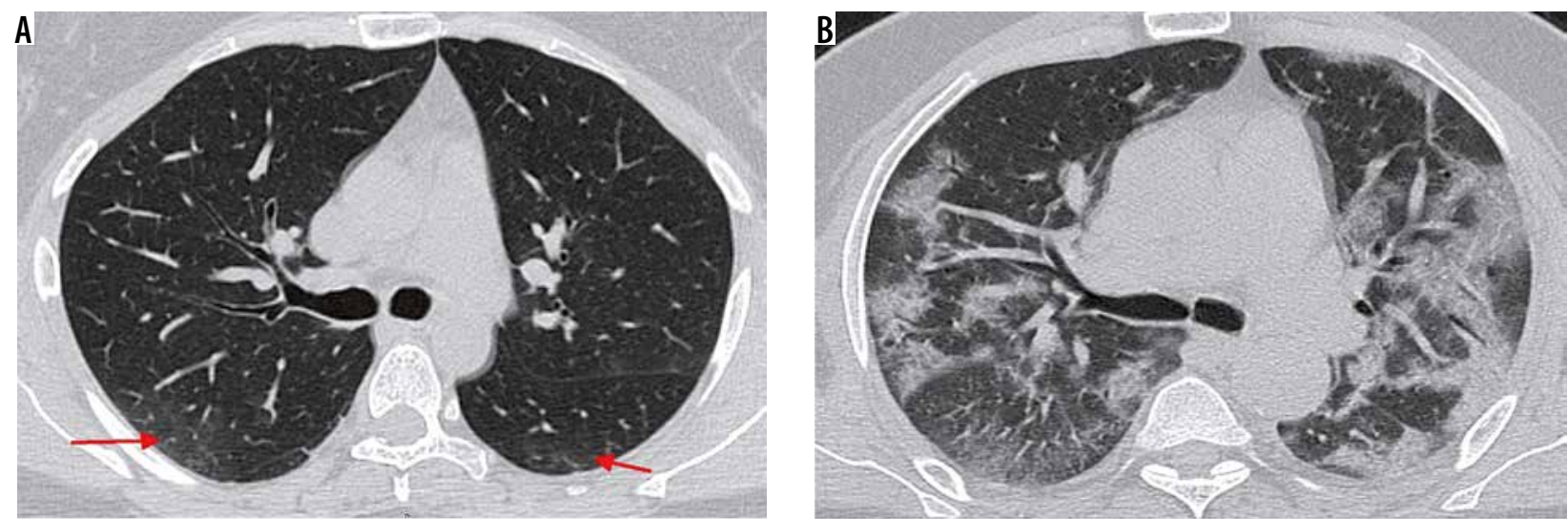

Figure 12. A 38-year-old diabetic female presented with two days of cough, fever, and breathlessness. A) Initial high-resolution computed tomography chest shows subtle ground-glass opacities (GGOs) in both lower lobes (red arrows). However, her condition in the ward worsened over the next 10 days. A repeat scan (B) shows multifocal dense GGOs with forming consolidations in both lung fields suggestive of severe stage of COVID-19 pneumonia 

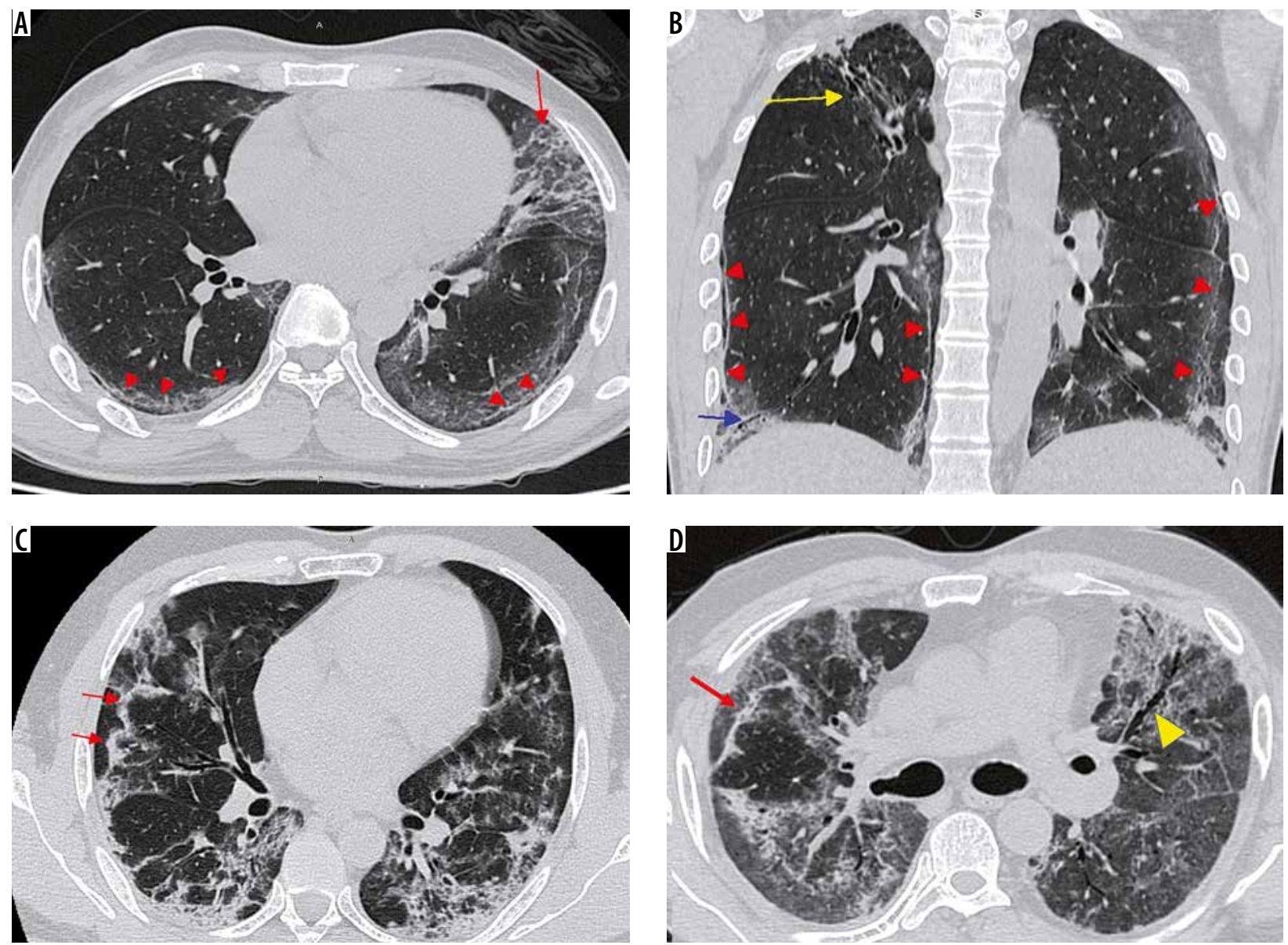

Figure 13. Typical findings of dissipative stage of COVID-19 pneumonia on high-resolution computed tomography chest. A-B) A 47-year-old man with symptoms for 12 days. Axial (A) and coronal (B) chest computed tomography (CT) images show curvilinear fibrotic lines parallel to the chest wall in both lung fields (red arrowheads) with extensive reticulations in the upper lingula (red arrow) and traction bronchiectasis (blue arrow). Also note fibrotic strands with adjacent pleural thickening and bronchiectasis in the right upper lobe (yellow arrow) as a sequalae to prior tubercular infection. C) Axial (T chest image in a 42-year-old female shows curvilinear opacities parallel to the chest wall (red arrows) with adjacent reticulations. D) Similar findings are also seen in another patient in dissipative stage of the disease (red arrow). Also note associated traction bronchiectasis in the left upper lobe (yellow arrowhead)

Subpleural curvilinear lines and fibrosis: Fine curvilinear opacities paralleling the pleural surfaces are seen in COVID-19 pneumonia which is related to fibrosis. Other fibrotic stripes may result due to scarring from chronic inflammation. Some authors believe the appearance of fibrosis is a good prognostic sign in the disease, indicating stabilisation of the disease status $[18,25]$. However, it may progress to interstitial lung disease subsequently.

Patients in advanced or severe stages of the disease can progress to dissipation stage with residual fibrosis [25]. This temporal evolution is best evaluated on chest HRCT (Figure 14).

\section{COVID-19 Reporting and Data System (CO-RADS)}

The "COVID-19 Standardised Reporting" working group of the Dutch Radiological Society has introduced CORADS as a standardised HRCT chest-based assessment scheme for suspected COVID-19 patients in similar lines to the systems used for breast and lung malignancies [26]. CO-RADS measures the suspicion for COVID-19 pneumo- nia using chest HRCT on a scale from 1 (very low probability) to 5 (very high probability) as well as CO-RADS 0 for technically inappropriate studies and CO-RADS 6 for RTPCR-proven SARS CoV-2 infection. The CO-RADS categories with associated findings are summarised in Table 1 , examples of which have been depicted in Figure 15.

\section{Total Severity Score}

Visual quantification of lung inflammation and correlation with clinical severity of the disease were performed by Li et al. [27]. In their study, patients were classified into 3 subgroups based on the clinical severity: mild (minimally symptomatic patients with normal CT), common, and severe-critical (patients with CT findings of COVID-19 and varying clinical manifestations). A CT visual quantitative score was devised by evaluating lung inflammation involving each lobe and summing up the scores of all five lobes. Each lobe was assessed for the percentage of lobar involvement: none (0\%), minimal (1-25\%), mild (26$50 \%)$, moderate (51-75\%), and severe (76-100\%), which 

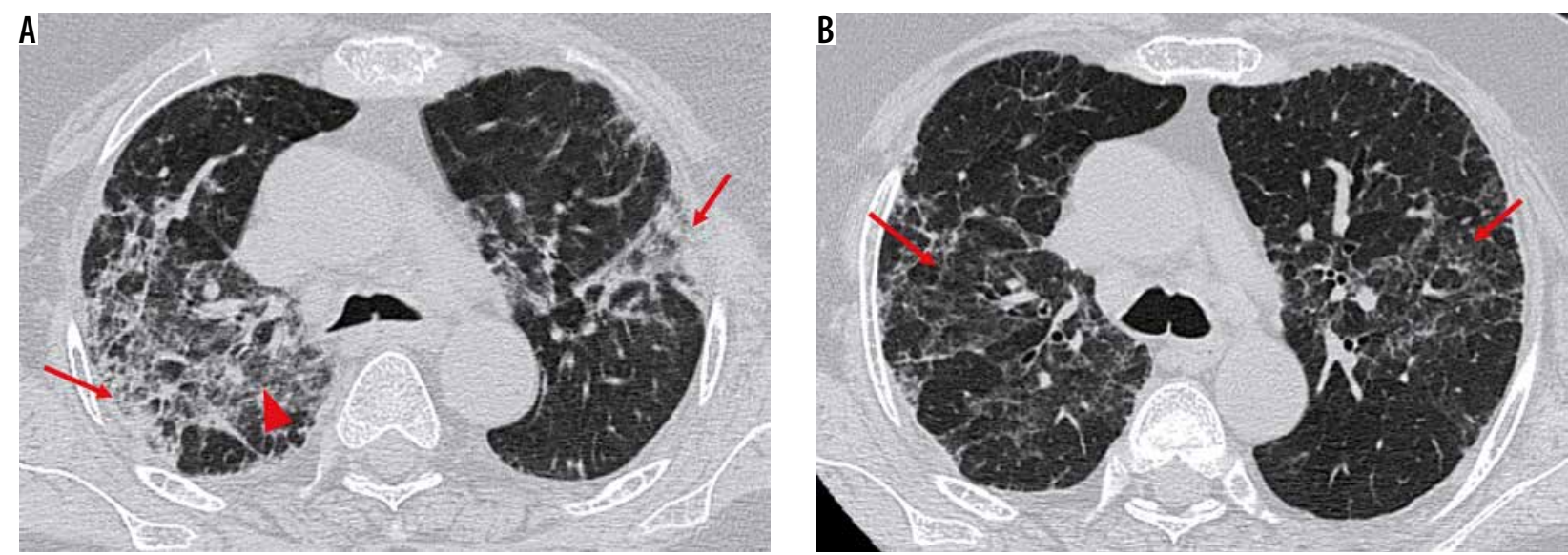

Figure 14. Temporal changes in a 55-year-old man with COVID-19 pneumonia on chest high-resolution computed tomography. A) Axial computed tomography (CT) chest images shows ground-glass opacities (GGOs) (red arrowhead) with interlobular septal thickening and formation of consolidations in both lung fields (red arrow). B) Follow-up chest CT performed after 1 month shows reduction in GGOs as compared to (A) with residual fibrosis (red arrows), suggesting progression to dissipation stage

Table 1. CO-RADS score

\begin{tabular}{|c|c|c|c|}
\hline $\begin{array}{l}\text { CO-RADS } \\
\text { score }\end{array}$ & $\begin{array}{l}\text { Chance of infection } \\
\text { with COVID-19 }\end{array}$ & CT findings & Alternate diagnosis suspected \\
\hline CO-RADS 0 & Not interpretable & $\begin{array}{l}\text { Incomplete or inadequate quality scans due to breathing } \\
\text { artefacts. }\end{array}$ & Scan technically insufficient, repeat study. \\
\hline CO-RADS 1 & Highly unlikely & $\begin{array}{l}\text { Normal CT scan, mild or severe emphysema, perifissural } \\
\text { nodules, lung tumour, or fibrosis }\end{array}$ & $\begin{array}{c}\text { Normal, non-infectious diseases like sarcoidosis, } \\
\text { congestive heart failure, malignancy, interstitial } \\
\text { lung disease to be considered. }\end{array}$ \\
\hline CO-RADS 2 & Unlikely & $\begin{array}{c}\text { A centrilobular nodular pattern (tree-in-bud sign), } \\
\text { bronchiectasis, segmental consolidation, and lung } \\
\text { cavitation. }\end{array}$ & $\begin{array}{l}\text { Infective aetiology not compatible with } \\
\text { COVID-19-like bronchitis, bronchiolitis, } \\
\text { pneumonia, or lung abscess. }\end{array}$ \\
\hline CO-RADS 3 & Equivocal & $\begin{array}{l}\text { Perihilar } \mathrm{GGO} \text {, extensive homogenous } \mathrm{GGO} \text {, consolidation } \\
\text { compatible with organising pneumonia. }\end{array}$ & $\begin{array}{l}\text { CT features compatible with COVID-19 } \\
\text { pneumonia, but other diseases like organising } \\
\text { pneumonia to be considered. }\end{array}$ \\
\hline CO-RADS 4 & Possible & $\begin{array}{l}\text { Findings similar to CO-RADS 5, but unilateral, not reaching } \\
\text { the visceral pleura, predominantly peri-bronchovascular } \\
\text { or superimposed on pre-existing abnormalities. }\end{array}$ & Highly suspicious for COVID-19. \\
\hline CO-RADS 5 & Highly likely & $\begin{array}{c}\text { GG0 with or without consolidation close to visceral pleura; } \\
\text { multifocal in distribution; sharply demarcated GG0 with } \\
\text { crazy-paving appearance, reverse halo sign, and vascular } \\
\text { thickening }\end{array}$ & CT features typical for COVID-19 infection. \\
\hline CO-RADS 6 & $P C R$ proven & - & SARS CoV-2 positive on RT-PCR. \\
\hline
\end{tabular}

corresponded to scores of $0,1,2,3$, and 4 , respectively. The score called the Total Severity Score (TSS) was obtained by adding up the severity scores of all the 5 lobes (ranging from 0 to 20). TSS showed a significant difference between the common type and severe-critical type patients with a cut-off of 7.5 having a high sensitivity and specificity [27]. Calculation of TSS score in a prototype case are shown in Figure 16.

\section{Association with pulmonary thromboembolism (Figure 17)}

Thrombotic complications of COVID-19 infection causing significant morbidity and mortality have recently been recognised in published literature [28-30]. Various manifestations such as deep venous thrombosis, pulmonary embolism, ischaemic stroke, and acute myocardial infarction have been reported. The pathophysiology has been ascribed to endothelial injury, inflammation, and microvascular thrombosis [30].

\section{Atypical imaging features (Figure 18)}

The presence of pulmonary nodules, mediastinal lymphadenopathy, and pleural effusions is rare in patients with COVID-19 [31]. At our institution, we have occasionally come across COVID-19 patients with enlarged 

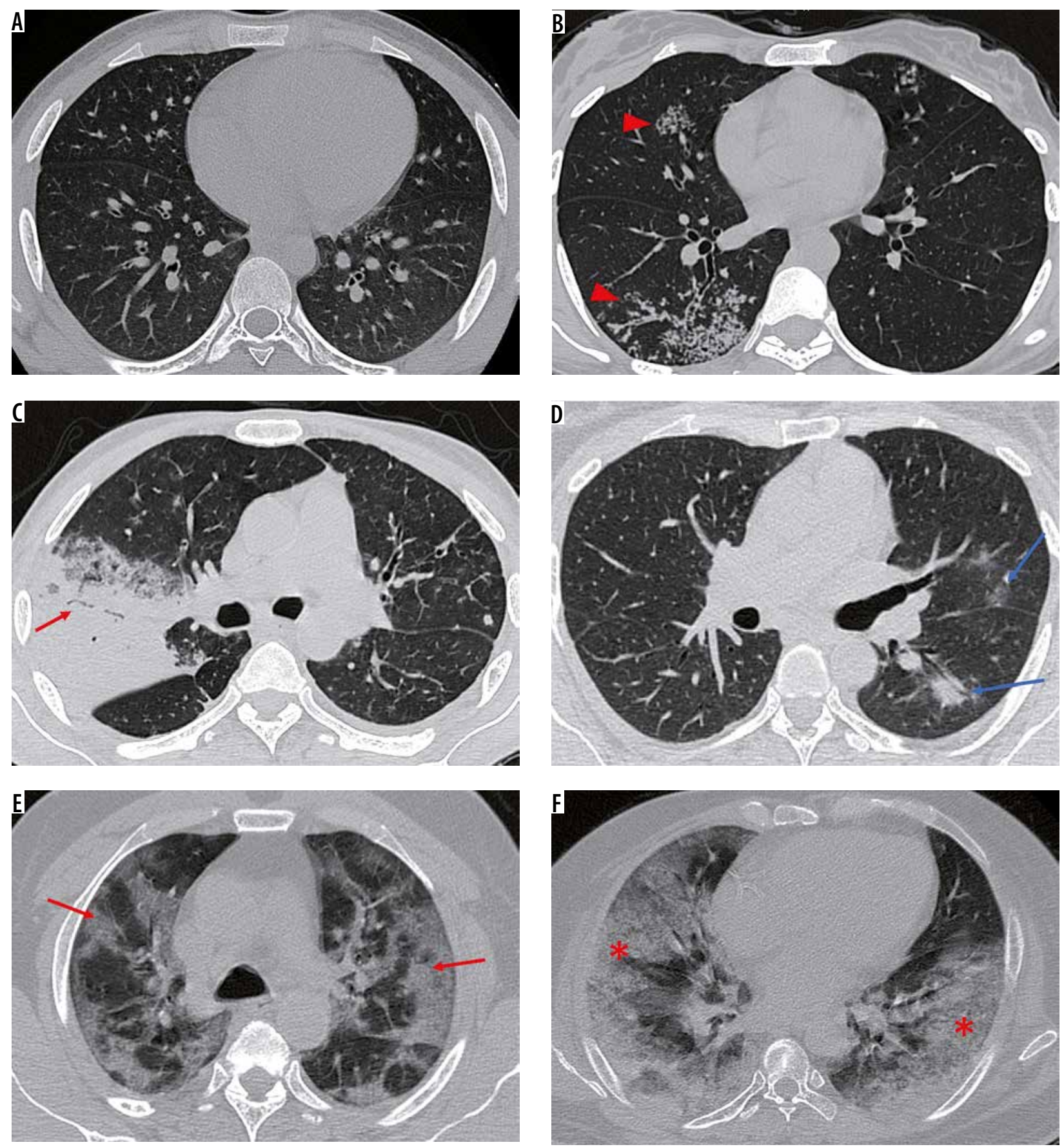

Figure 15. Representative axial high-resolution computed tomography (HRCT) chest images depicting CO-RADS. A) Normal HRCT chest: C0-RADS 1. B) Centrilobular nodules with tree-in-bud configuration in the right lung (red arrowheads) - bronchiolitis, later diagnosed as active pulmonary tuberculosis. CO-RADS 2. C) Dense consolidation with air bronchogram (red arrow) and surrounding ground-glass opacity (GG0) in a patient with Klebsiella pneumonia: CO-RADS 3. D) Peribronchovascular GGOs in the left lung (blue arrows): (0-RADS 4. Nasopharyngeal swab was positive for SARS-CoV-2. E) Peripheral and subpleural predominant multifocal GGOs with interlobular septal thickening - crazy-paving appearance (red arrows) in both lungs: CO-RADS 5. This was later proven to be COVID-19 pneumonia. F) Extensive, peripheral confluent bilateral GGOs with septal thickening in a patient with acute respiratory distress syndrome secondary to COVID-19 pneumonia

mediastinal lymph nodes that could not be attributed to any other pathology (Figure 18A). A lymph node is classified as enlarged if it measures more than $10 \mathrm{~mm}$ in the short axis. Rare cases of COVID-19-positive patients with subsolid pulmonary nodules have also been observed (Figure 18C-D).
The typical mild COVID-19 pneumonia usually starts as a small subpleural, unilateral, or bilateral GGO in the lower lobes. This then develops into the crazy-paving pattern and subsequent consolidation, with an ARDSlike imaging pattern in severe cases. The lesions gradually resolve with residual GGO and subpleural parenchymal 

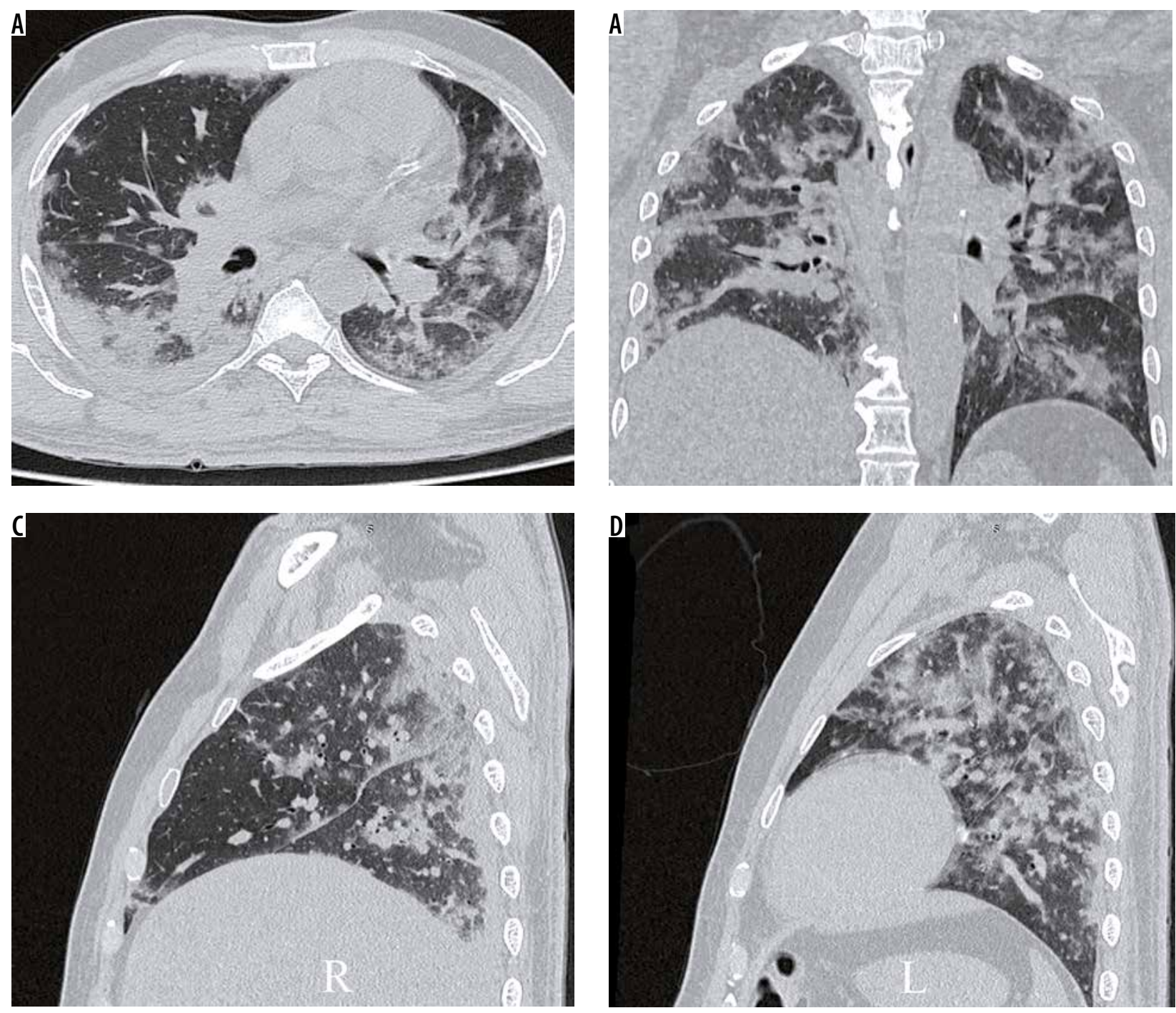

Figure 16. Total Severity Score (TSS) calculation in COVID-19 pneumonia using high-resolution computed tomography chest. Axial (A) and coronal (B) chest computed tomography (CT) images in a 42-year-old man with clinically severe COVID-19 pneumonia shows multifocal peripheral ground-glass opacities and consolidations in both lungs. Sagittal reformatted images of the right lung (C) and left lung (D) show less than $25 \%$ involvement of right middle lobe (score 1); right upper and both lower lobes have a score of 2 each, and left upper lobe has a score of 3 . The TSS in this patient was 10, correlating with the clinical severity
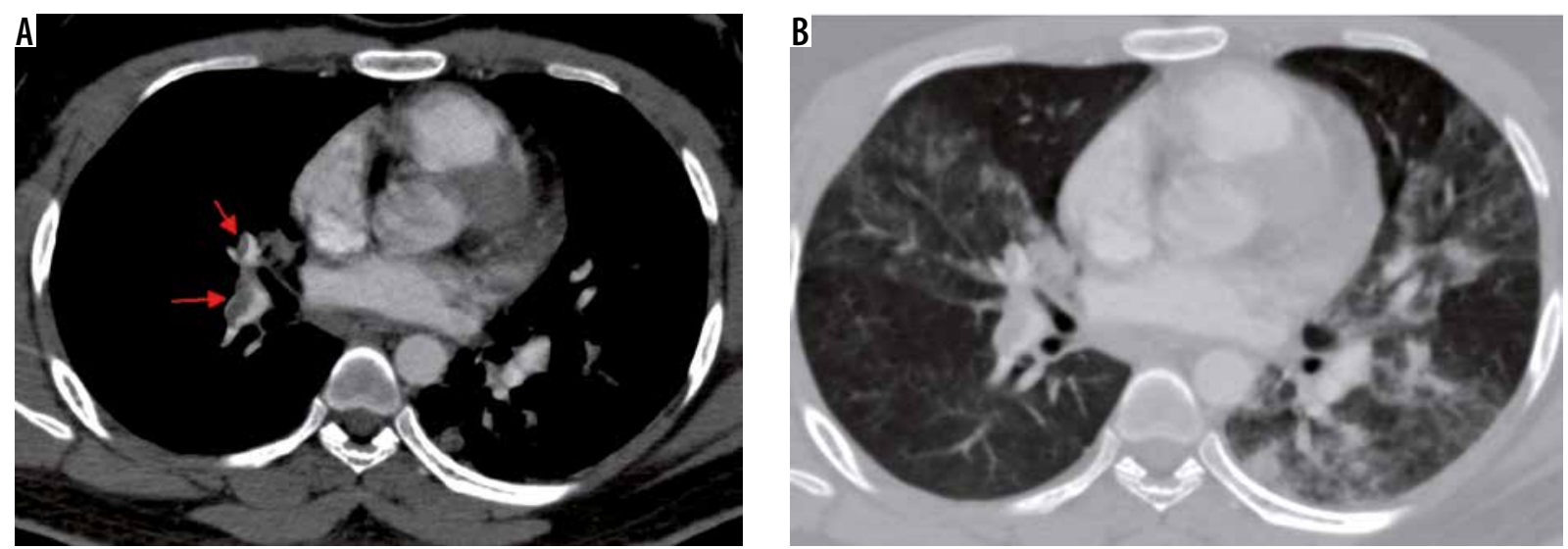

Figure 17. A 25-year-old man with COVID-19 pneumonia presenting with sudden onset breathlessness. A) Computed tomography (CT) pulmonary angiogram image shows an eccentric partial thrombus in the right descending pulmonary artery extending into the right middle lobe branch (red arrows). B) Axial CT image with lung window at the same level shows multifocal ground-glass opacities in both lung fields 

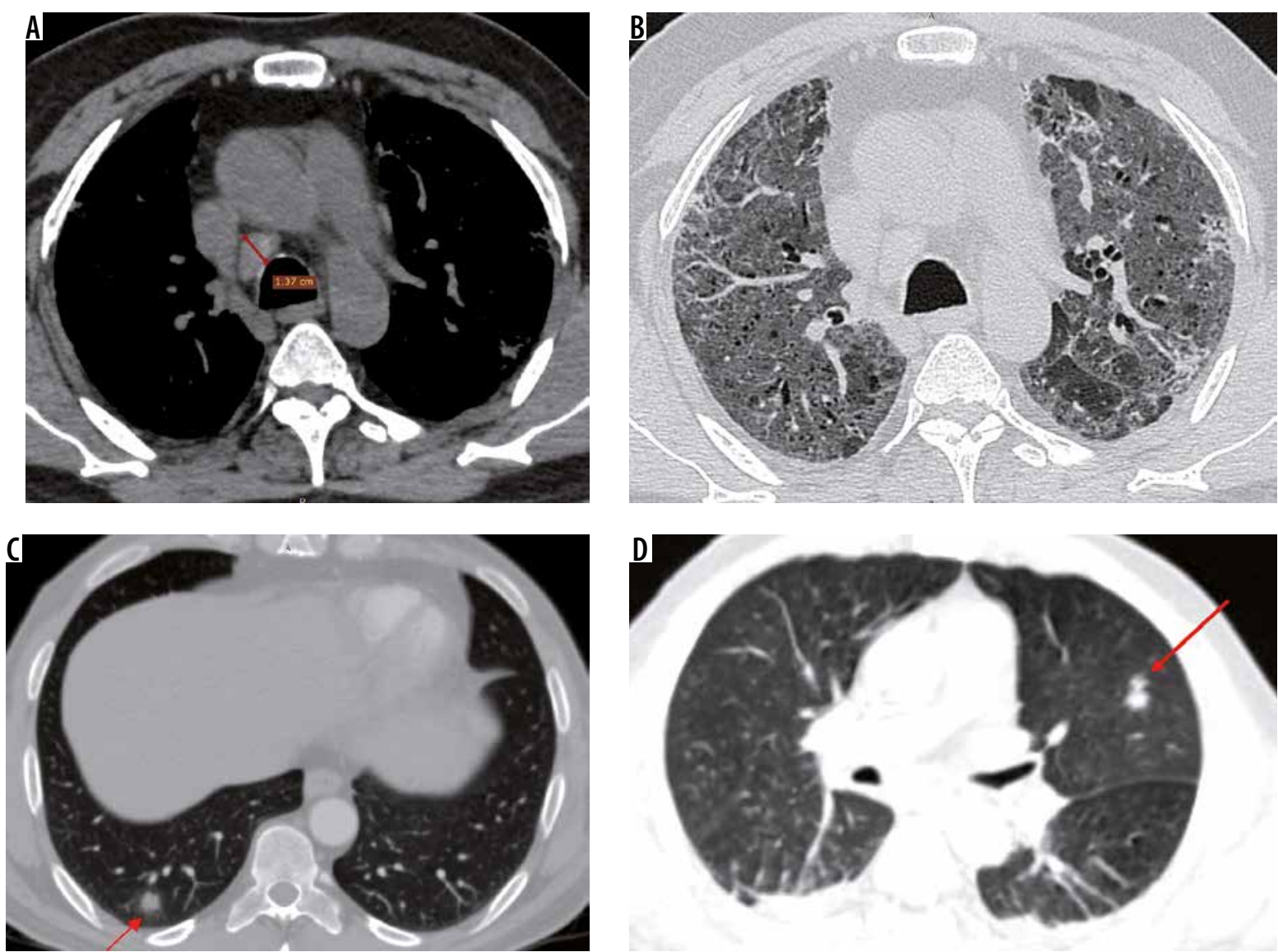

Figure 18. Atypical computed tomography (CT) chest findings in COVID-19 pneumonia. A) Axial chest CT image in a 32-year-old man in dissipative stage of disease shows an enlarged right paratracheal lymph node. B) Axial high-resolution computed tomography chest image in the same patient shows interlobular septal thickening with traction bronchiectasis and ground glass opacities suggestive of progression to interstitial lung disease. C-D) Axial chest CT images in 2 different patients with COVID-19 pneumonia show small subsolid nodules (red arrows)

bands over two weeks. This temporal evolution of the disease is illustrated in Figure 19.

\section{Differential diagnosis}

Viruses are an important cause of respiratory tract infections, with overlapping clinical features and imaging findings. Although the imaging appearance of COVID-19 is similar to that of other viruses like influenza, parainfluenza virus, and respiratory syncytial virus, significant differences do exist. Both human parainfluenza and influenza viruses show centrilobular nodules in addition to GGOs and consolidations [14]. Because severe acute respiratory syndrome (SARS) and MERS belong to the same coronavirus family, they share clinical features and imaging findings with COVID-19 pneumonia. Peripheral multifocal air space opacities are seen in all the 3 viral pneumonias; however, SARS has a greater incidence of focal unilateral involvement in the early course of disease, while both COVID-19 and MERS are predominantly bi- lateral. MERS pneumonia has a higher incidence of pleural effusions and upper-lobe involvement as compared to COVID-19 pneumonias [32]. A final diagnosis is often made with laboratory analysis and virus isolation.

\section{Conclusions}

COVID-19 has affected every country throughout the world due to its contagious nature. Even though a large segment of the population has been affected, the fatality rate is higher in people with comorbidities. In the fight against this pandemic, radiologists play a crucial role in the rapid identification and early diagnosis of suspected patients. Thorough knowledge of the imaging features of COVID-19 pneumonia can allow for early containment and prompt response to help overcome this pandemic.

\section{Conflict of interest}

The authors report no conflict of interest. 


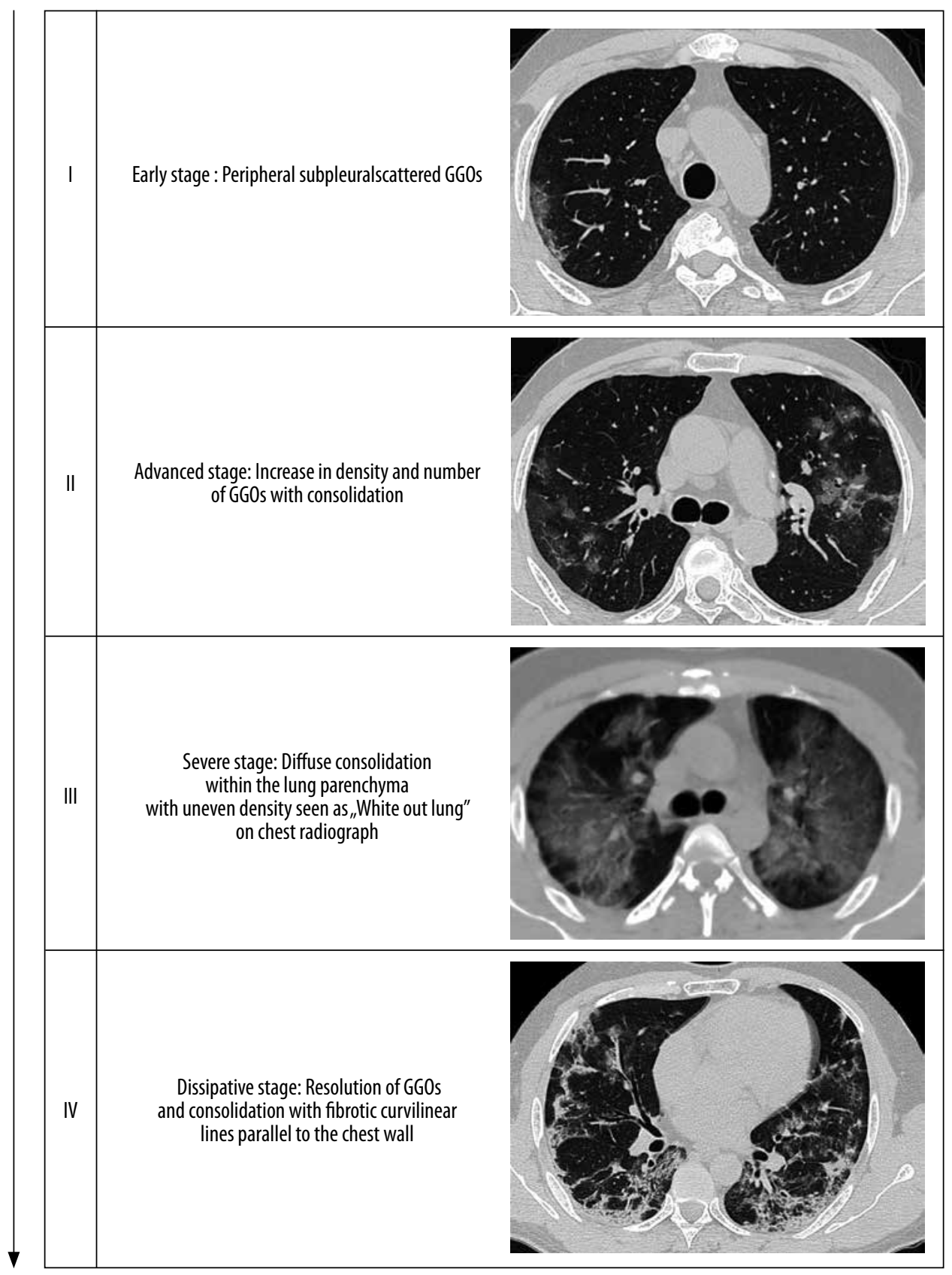

Figure 19. Different stages of COVID-19 pneumonia on chest high-resolution computed tomography

\section{References}

1. Zhu N, Zhang D, Wang W, et al. A novel coronavirus from patients with pneumonia in China, 2019. N Engl J Med 2020; 382: 727-733.

2. Wang D, Hu B, Hu C, et al. Clinical characteristics of 138 hospitalized patients with 2019 novel coronavirus-infected pneumonia in Wuhan, China. JAMA 2020; 323: 1061-1069.

3. Spinato G, Fabbris C, Polesel J, et al. Alterations in smell or taste in mildly symptomatic outpatients with SARS-CoV-2 infection. JAMA 2020; 323: 2089-2090.

4. Lee DW, Gardner R, Porter DL, et al. Current concepts in the diagnosis and management of cytokine release syndrome. Blood 2014; 124: 188-195.

5. Tan W, Zhao X, Ma X, et al. A novel coronavirus genome identified in a cluster of pneumonia cases - Wuhan, China 2019-2020. China CDC Weekly 2020; 2: 61-62.
6. Pan $Y$, Guan $H$, Zhou S, et al. Initial CT findings and temporal changes in patients with the novel coronavirus pneumonia (2019-nCoV): a study of 63 patients in Wuhan, China. Eur Radiol 2020; 30: 3306-3309.

7. Jacobi A, Chung M, Bernheim A, et al. Portable chest X-ray in coronavirus disease-19 (COVID-19): a pictorial review. Clin Imag 2020; 64: 35-42.

8. Wong HYF, Lam HYS, Fong AH, et al. Frequency and distribution of chest radiographic findings in COVID-19 positive patients. Radiology 2020; 296: E72-E78.

9. Yoon SH, Lee KH, Kim JY, et al. Chest radiographic and CT findings of the 2019 novel coronavirus disease (COVID-19): analysis of nine patients treated in Korea. Korean J Radiol 2020; 21: 494-500.

10. Peng QY, Wang XT, Zhang LN, et al. Findings of lung ultrasonography of novel corona virus pneumonia during the 2019-2020 epidemic. Intensive Care Med 2020; 46: 849-850. 
11. Huang Y, Wang S, Liu Y, et al. A preliminary study on the ultrasonic manifestations of peripulmonary lesions of non-critical novel coronavirus pneumonia (COVID-19). SSRN. 2020. doi: https://dx.doi. org/10.2139/ssrn.3544750 [In press].

12. Xu X, Chen P, Wang J, et al. Evolution of the novel coronavirus from the ongoing Wuhan outbreak and modeling of its spike protein for risk of human transmission. Sci China Life Sci 2020; 63: 457-460.

13. Wang Y, Dang C, Hu Y, et al. Temporal changes of CT findings in 90 patients with COVID-19 pneumonia: a longitudinal study. Radiology 2020; 296: E55-E64.

14. Yang W, Sirajuddin A, Zhang X, et al. The role of imaging in 2019 novel coronavirus pneumonia (COVID-19). Eur Radiol 2020. doi: 10.1007/s00330-020-06827-4 [In press].

15. Hansell DM, Bankier AA, MacMahon H, et al. Fleischner Society: glossary of terms for thoracic imaging. Radiology 2008; 246: 697-722.

16. Chung M, Bernheim A, Mei X, et al. CT imaging features of 2019 novel coronavirus (2019-nCoV). Radiology 2020; 295: 202-207.

17. Kunhua Li JW, Wu F, Guo D, et al. The clinical and chest CT features associated with severe and critical COVID-19 pneumonia. Invest Radiol 2020. doi: 10.1097/RLI.0000000000000672 [In press].

18. Pan F, Ye T, Sun P, et al. Time course of lung changes on chest CT during recovery from 2019 novel coronavirus (COVID-19) pneumonia. Radiology 2020; 295: 715-721.

19. Xu Z, Shi L, Wang Y, et al. Pathological findings of COVID-19 associated with acute respiratory distress syndrome. Lancet Respir Med 2020; 8: 420-422.

20. Zou S, Zhu X. FDG PET/CT of COVID-19. Radiology 2020. doi: https://doi.org/10.1148/radiol.2020200770 [In press].

21. Qin C, Liu F, Yen TC, Lan X. 18F-FDG PET/CT findings of COVID-19: a series of four highly suspected cases. Eur J Nucl Med Mol Imaging 2020; 47: 1281-1286.

22. Pinto PS. The CT halo sign. Radiology 2004; 230 : 109-110.
23. Shi H, Han X, Jiang N, et al. Radiological findings from 81 patients with COVID-19 pneumonia in Wuhan, China: a descriptive study. Lancet Infect Dis 2020; 20: 425-434.

24. Fang Y, Zhang H, Xu Y, et al. CT manifestations of two cases of 2019 novel coronavirus (2019-nCoV) pneumonia. Radiology 2020; 295: 208-209.

25. Lei P, Fan B, Mao J, et al. The progression of computed tomographic (CT) images in patients with coronavirus disease (COVID-19) pneumonia. J Infect 2020; 80: 30-31.

26. Prokop M, van Everdingen W, van Rees Vellinga T, et al. CO-RADS - a categorical CT assessment scheme for patients with suspected COVID-19: definition and evaluation. Radiology 2020; 296: E97-E104.

27. Li K, Fang Y, Li W, et al. CT image visual quantitative evaluation and clinical classification of coronavirus disease (COVID-19). Eur Radiol 2020; 30: 4407-4416.

28. Poyiadi N, Cormier P, Patel PY, et al. Acute pulmonary embolism and COVID-19. Radiology 2020; 297: E335-E338.

29. Bikdeli B, Madhavan MV, Jimenez D, et al. COVID-19 and thrombotic or thromboembolic disease: implications for prevention, antithrombotic therapy, and follow-up: JACC state-of-the-art review. J Am Coll Radiol 2020; 75: 2950-2973.

30. Klok FA, Kruip MJHA, Van der Meer NJM, et al. Incidence of thrombotic complications in critically ill ICU patients with COVID-19. Thromb Res 2020; 191: 145-147.

31. Carotti M, Salaffi F, Sarzi-Puttini P, et al. Chest CT features of coronavirus disease 2019 (COVID-19) pneumonia: key points for radiologists. Radiol Med 2020; 125: 636-646.

32. Hosseiny M, Kooraki S, Gholamrezanezhad A, et al. Radiology perspective of coronavirus disease 2019 (COVID-19): lessons from severe acute respiratory syndrome and middle east respiratory syndrome. AJR Am J Roentgenol 2020; 214: 1078-1082. 